\title{
The Grassmannian origin of dual superconformal invariance
}

\author{
Nima Arkani-Hamed, ${ }^{a}$ Freddy Cachazo $^{b}$ and Clifford Cheung ${ }^{c, d}$ \\ ${ }^{a}$ School of Natural Sciences, Institute for Advanced Study, \\ Princeton, NJ 08540, U.S.A. \\ ${ }^{b}$ Perimeter Institute for Theoretical Physics, \\ Waterloo, Ontario N2J W29, Canada \\ ${ }^{c}$ Berkeley Center for Theoretical Physics, University of California Berkeley, \\ Berkeley, CA 94720, U.S.A. \\ ${ }^{d}$ Theoretical Physics Group, Lawrence Berkeley National Laboratory, \\ Berkeley, CA 94720, U.S.A. \\ E-mail: arkani@ias.edu , fcachazo@perimeterinstitute.ca, \\ clifford. cheung@berkeley.edu
}

ABSTRACT: A dual formulation of the $\mathrm{S}$ Matrix for $\mathcal{N}=4 \mathrm{SYM}$ has recently been presented, where all leading singularities of $n$-particle $\mathrm{N}^{k-2} \mathrm{MHV}$ amplitudes are given as an integral over the Grassmannian $G(k, n)$, with cyclic symmetry, parity and superconformal invariance manifest. In this short note we show that the dual superconformal invariance of this object is also manifest. The geometry naturally suggests a partial integration and simple change of variable to an integral over $G(k-2, n)$. This change of variable precisely corresponds to the mapping between usual momentum variables and the "momentum twistors" introduced by Hodges, and yields an elementary derivation of the momentumtwistor space formula very recently presented by Mason and Skinner, which is manifestly dual superconformal invariant. Thus the $G(k, n)$ Grassmannian formulation allows a direct understanding of all the important symmetries of $\mathcal{N}=4$ SYM scattering amplitudes.

KeYwords: Supersymmetric gauge theory, Duality in Gauge Field Theories

ARXIV EPRINT: 0909.0483 
Recently a simple formula has been conjectured for all leading singularities of $\mathcal{N}=4$ SYM amplitudes [1]. Working in twistor space, the leading singularities of single trace, color stripped, $n$-particle $\mathrm{N}^{k-2} \mathrm{MHV}$ amplitudes are associated with the object

$$
\mathcal{L}_{n ; k}=\frac{1}{\operatorname{vol}(\mathrm{GL}(k))} \int \frac{d^{k \times n} C_{\alpha a}}{\left(C_{1} C_{2} \cdots C_{k}\right) \cdots\left(C_{n} C_{1} \cdots C_{k-1}\right)} \prod_{\alpha=1}^{k} \delta^{4 \mid 4}\left(C_{\alpha a} \mathcal{W}_{a}\right)
$$

where

$$
\left(C_{m_{1}} \cdots C_{m_{k}}\right)=\epsilon^{\alpha_{1} \cdots \alpha_{k}} C_{\alpha_{1} m_{1}} \cdots C_{\alpha_{k} m_{k}}
$$

are minors of the $k \times n$ matrix $C$. Here $a=1, \cdots, n$ labels the $n$ external particles. The integrand has a $\operatorname{GL}(k)$ "gauge symmetry" under which $C_{\alpha a} \rightarrow L_{\alpha}^{\beta} C_{\beta a}$ for any $k \times k$ matrix $L$, and so we have to "gauge-fix" by dividing by $\operatorname{vol}(\mathrm{GL}(k))$.

Going back to momentum space, the integral turns into a multi-dimensional contour integral. In [1], substantial evidence was given that the residues of the integrand provide a basis for constructing tree amplitudes as well as 1- and 2-loop leading singularities. Multi-dimensional residue theorems give rise to a large number of remarkable identities between the residues, which guarantee the equivalence of many different representations of the same amplitude and enforce the cancelation of non-local poles as well as consistent infrared structure at loop level. In [1], it was also shown that the action of cyclic, parity and superconformal symmetries on eq. (1) is manifest. In this short note we show that the dual superconformal invariance [2-4] of eq. (1) is also manifest.

Let us immediately transform eq. (1) back into momentum space

$$
\mathcal{L}_{n ; k}=\frac{1}{\operatorname{vol}(\mathrm{GL}(k))} \int \frac{d^{k \times n} C_{\alpha a}}{\left(C_{1} C_{2} \cdots C_{k}\right) \cdots\left(C_{n} \cdots C_{k-1}\right)} \prod_{\alpha} \delta^{4}\left(C_{\alpha a} \tilde{\eta}_{a}\right) \delta^{2}\left(C_{\alpha a} \tilde{\lambda}_{a}\right) \int d^{2} \rho_{\alpha} \delta^{2}\left(\lambda_{a}-C_{\alpha a} \rho_{\alpha}\right)
$$

In this form the geometric character of the integral over $C$, already discussed in [1], is completely clear. The $\lambda_{a}, \tilde{\lambda}_{a}$ are 2 -planes in the $n$-dimensional space, while $C$ is a $k$ plane. The integral is then over the space of $k$-planes in $n$-dimensions - the Grassmannian $G(k, n)$. The first set of bosonic delta functions forces $C$ to be orthogonal to the $\tilde{\lambda}$ plane, while the second forces some linear combination of the $k n$-vectors in $C$ to equal $\lambda$; in other words, the $k$-plane $C$ must also contain the 2-plane $\lambda$. Note that these two requirements can be satisfied only if the $\lambda$ plane is orthogonal to the $\tilde{\lambda}$ plane $-\sum \lambda_{a} \tilde{\lambda}_{a}=0-$ which is nothing other than the statement of momentum conservation. ${ }^{1}$

Given that the $C$ plane must contain the $\lambda$ plane, it is very natural to split $C$ into a part that literally is the $\lambda$ 2-plane, and only perform the integral over the remaining $(k-2)$ directions. This motivates us to try and write the resulting expression as an integral over $G(k-2, n)$. This is essentially trivial - the only (minor) complication is that the minors appearing in eq. (1) are $k \times k$ minors, not $(k-2) \times(k-2)$ minors. But from high-school algebra we are familiar with a natural linear transformation which

\footnotetext{
${ }^{1}$ Note that in writing eq. (1) we are working with real variables in $(2,2)$ signature, and in the $n$ dimensional space there is a natural notion of "orthogonality" associated with the standard dot product. As discussed in [1], the final, fully gauge-fixed form of the expression for $\mathcal{L}_{n ; k}$ is defined for arbitrary complex momenta.
} 
maps the larger minors to the smaller minors. As we will see this linear transformation takes us from the momentum variables to the "momentum twistor" variables recently introduced in a remarkable paper by Hodges [5]. The remaining integral over $G(k-2, n)$ precisely yields the momentum-twistor Grassmannian formula very recently presented by Mason and Skinner [6]. This expression is manifestly dual superconformal invariant. In this way momentum twistors and dual superconformal invariance are seen to follow very naturally from the Grassmannian formulation, and we see that eq. (1) provides a simple understanding of all the important symmetries of the theory.

To get started, let us turn to "gauge-fixing" the GL $(k)$ symmetry, which we can do by introducing a gauge-fixing function $\Delta_{\mathrm{GL}(\mathrm{k})}$ which can in general be a function of $C_{\alpha a}$ and $\rho_{\alpha}$, and writing

$$
\mathcal{L}_{n ; k}=\int \frac{d^{k \times n} C_{\alpha a} \Delta_{\mathrm{GL}(k)}}{\left(C_{1} C_{2} \cdots C_{k}\right) \cdots\left(C_{n} C_{1} \cdots C_{k-1}\right)} \prod_{\alpha} \delta^{4}\left(C_{\alpha a} \tilde{\eta}_{a}\right) \delta^{2}\left(C_{\alpha a} \tilde{\lambda}_{a}\right) \int d^{2} \rho_{\alpha} \delta^{2}\left(\lambda_{a}-C_{\alpha a} \rho_{\alpha}\right)
$$

In practice, we can choose $\Delta_{\mathrm{GL}(k)}$ to be a product of $k^{2}$ delta functions fixing some components of $C, \rho$ to some canonical values. For instance in [1], we implicitly chose $\Delta$ to only depend on $C$, and used it to fix $k$ of the columns of $C$ to an orthonormal basis. This gauge-fixing was convenient for showing the parity invariance of $\mathcal{L}_{n ; k}$, as well as making the connection between eq. (1) and the "link representation" of [7]. In this note we will find another gauge-fixing convenient, the analog of which was already used in [1] in the discussion of MHV amplitudes.

Since our ultimate goal is to be left with an integral over $k-2$ planes, we wish to start by only partially gauge-fixing, leaving a $\mathrm{GL}(k-2)$ to be fixed only at the end. Therefore, we start by imposing $k^{2}-(k-2)^{2}=4 k-4=2 k+2(k-2)$ conditions. In other words we will write

$$
\Delta_{\mathrm{GL}(k)}=\Delta_{\mathrm{GL}(k) \rightarrow \mathrm{GL}(k-2) \times T_{2}} \times \Delta_{\mathrm{GL}(k-2) \times T_{2} \rightarrow \mathrm{GL}(k-2)} \times \Delta_{\mathrm{GL}(k-2)}
$$

which gauge fix the GL $(k)$ in steps, first down to $\operatorname{GL}(k-2) \times T_{2}$ where $T_{2}$ are translations by a vector in the $\lambda$ plane, then to $\operatorname{GL}(k-2)$. In the first step, we use $2 k$ delta functions to gauge fix the two $k$-vectors $\rho$ to

$$
\rho=\left(\begin{array}{cc}
1 & 0 \\
0 & 1 \\
0 & 0 \\
\vdots & \vdots \\
0 & 0
\end{array}\right)
$$

Formally we choose

$$
\Delta_{\mathrm{GL}(k) \rightarrow \mathrm{GL}(k-2) \times T_{2}}=\prod_{\alpha} \delta^{2}\left(\rho_{\bar{\alpha} \alpha}-\delta_{\bar{\alpha} \alpha}\right) .
$$

Here we use $\bar{\alpha}=1,2$ to label the spinor Lorentz indices. These equation fix all the $\rho$ variables; the $\delta^{2}\left(\lambda_{a}-C_{\alpha a} \rho_{\alpha}\right)$ in the integral then fixes the top two rows of $C$ to be the $\lambda$ 
2-plane, that is $C_{\bar{\alpha} a}=\lambda_{\bar{\alpha} a}$, or

$$
C=\left(\begin{array}{cccc}
\lambda_{\bar{\alpha}=1,1} & \lambda_{\bar{\alpha}=1,2} & \cdots & \lambda_{\bar{\alpha}=1, n} \\
\lambda_{\bar{\alpha}=2,1} & \lambda_{\bar{\alpha}=2,2} & \cdots & \lambda_{\bar{\alpha}=2, n} \\
C_{3,1} & C_{3,2} & \cdots & C_{3, n} \\
\vdots & \vdots & \ddots & \vdots \\
C_{k, 1} & C_{k, 2} & \cdots & C_{k, n}
\end{array}\right)
$$

With this form we can factor out the momentum and super-momentum conserving delta functions trivially, since

$$
\prod_{\alpha=1}^{k} \delta^{4}\left(C_{\alpha a} \tilde{\eta}_{a}\right) \delta^{2}\left(C_{\alpha a} \tilde{\lambda}_{a}\right)=\delta^{4}\left(\lambda_{a} \tilde{\lambda}_{a}\right) \delta^{8}\left(\lambda_{a} \tilde{\eta}_{a}\right) \times \prod_{\hat{\alpha}=3}^{k} \delta^{4}\left(C_{\hat{\alpha} a} \tilde{\eta}_{a}\right) \delta^{2}\left(C_{\hat{\alpha} a} \tilde{\lambda}_{a}\right)
$$

where we use the index $\hat{\alpha}$ to run over the $(k-2)$ values $3,4, \cdots, k$.

Having done this, the unfixed part of $\mathrm{GL}(k)$ then consists of $\mathrm{GL}(k-2)$ transformations, together with translations $T_{2}$ of the remaining $(k-2) n$-vectors by any vector in the $\lambda$ plane. Geometrically, the most natural way to gauge fix $T_{2}$ is to impose the $2 \times(k-2)$ constraints that the remaining $(k-2)$ vectors in $C$ are orthogonal to the $\lambda$ plane:

$$
\Delta_{\mathrm{GL}(k-2) \times T_{2} \rightarrow \mathrm{GL}(k-2)}=J \prod_{\hat{\alpha}} \delta^{2}\left(C_{\hat{\alpha} a} \lambda_{a}\right)
$$

Here $J$ is some Jacobian that only depends on the $\lambda$ 's. Here we have used the index $\hat{\alpha}=3,4, \cdots, k$ to denote the remaining $(k-2)$ rows of $C$.

We have thus arrived at

$$
\mathcal{L}_{n ; k}=\frac{J \delta^{4}\left(\lambda_{a} \tilde{\lambda}_{a}\right) \delta^{8}\left(\lambda_{a} \tilde{\eta}_{a}\right)}{\operatorname{vol}(\mathrm{GL}(k-2))} \int \frac{d^{(k-2) \times n} C_{\hat{\alpha} a}}{\left(C_{1} C_{2} \cdots C_{k}\right) \cdots\left(C_{n} C_{1} \cdots C_{k-1}\right)} \prod_{\hat{\alpha}} \delta^{2}\left(C_{\hat{\alpha} a} \lambda_{a}\right) \delta^{2}\left(C_{\hat{\alpha} a} \tilde{\lambda}_{a}\right) \delta^{4}\left(C_{\hat{\alpha} a} \tilde{\eta}_{a}\right)
$$

This form is almost what we want: an integral manifestly over a space of $(k-2)$ planes in $n$ dimensions. ${ }^{2}$ However the problem is that the minors appearing in the denominator of the measure involve the full $k \times n$ matrix $C$, whose first two rows are the $\lambda$ plane. It is natural to try and find a linear transformation that maps the $k \times k$ minors to $(k-2) \times(k-2)$ minors. Consider the minor $\left(C_{1} C_{2} \cdots C_{k-1} C_{k}\right)$. We will take linear transformations of the columns of $C$, so that the first two rows of the middle $2, \cdots, k-1$ columns are set to zero. The simplest way to do this is to define new $k$ vectors $D_{\alpha b}$ as

$$
D_{\alpha b}=x_{b} C_{\alpha b-1}+y_{b} C_{\alpha b}+z_{b} C_{\alpha b+1}
$$

Clearly $D_{2}, \cdots, D_{k-1}$ are a linear combination of $C_{1}, \cdots, C_{k}$, therefore

$$
\left(C_{1} D_{2} \cdots D_{k-1} C_{k}\right)=f(x, y, z)\left(C_{1} \cdots C_{k}\right)
$$

\footnotetext{
${ }^{2}$ Note that for general $2 k>n, G(k-2, n)$ is not contained in $G(k, n)$. Geometrically, the space of $k$-planes that are forced to contain a given 2-plane is the same of the space of $(k-2)$ planes living in $(n-2)$ dimensions: $G(k-2, n-2)$ is contained in $G(k, n)$. The $\delta^{2}(C \cdot \lambda)$ factors in eqs. (10), (11) are reducing the integral over $G(k-2, n)$ to one over $G(k-2, n-2)$, but in a moment we will find these delta functions nicely unify with the remaining ones, and the resulting object is naturally integrated over $G(k-2, n)$.
} 
We choose $x, y, z$ so that $D_{\bar{\alpha} b}=0$. This fixes $D$ to be of the form

$$
D_{\alpha b}=q_{b}\left(\langle b+1 b\rangle C_{\alpha b-1}+\langle b-1 b+1\rangle C_{\alpha b}+\langle b b-1\rangle C_{\alpha b+1}\right)
$$

where $q_{b}$ is any normalization factor. Here $D_{\bar{\alpha} b}=0$ due to the Schouten identity $\langle b+1 b\rangle \lambda_{b-1}+\langle b-1 b+1\rangle \lambda_{b}+\langle b b-1\rangle \lambda_{b+1}=0$. Clearly

$$
\left(C_{1} D_{2} \cdots D_{k-1} C_{k}\right)=\left\langle\lambda_{1} \lambda_{k}\right\rangle\left(D_{2} \cdots D_{k-1}\right)
$$

where $\left(D_{2} \cdots D_{k-1}\right)$ is the $(k-2) \times(k-2)$ minor made from the non-zero elements $D_{\hat{\alpha} b}$ :

$$
\left(D_{2} \cdots D_{k-1}\right)=\epsilon^{\hat{\alpha}_{1} \cdots \hat{\alpha}_{k-2}} D_{\hat{\alpha}_{1} 2} \cdots D_{\hat{\alpha}_{k-2} k-1}
$$

Thus we have succeeded in mapping the $k \times k$ minors to $(k-2) \times(k-2)$ minors up to a $\lambda$ dependent factor

$$
\left(C_{1} \cdots C_{k}\right)=J^{\prime}\left(D_{2} \cdots D_{k-1}\right)
$$

What we have done is to introduce an $n \times n$ linear transformation $Q_{a b}$

$$
D_{\alpha b}=C_{\alpha a} Q_{a b}
$$

where

$$
Q_{a b}=q_{b} \times\left(\langle b-1 b+1\rangle \delta_{a b}+\langle b b-1\rangle \delta_{a, b+1}+\langle b+1 b\rangle \delta_{a, b-1}\right)
$$

satisfying

$$
\lambda_{a} Q_{a b}=0
$$

Now, using $Q$, we can re-write

$$
\tilde{\lambda}_{a}=Q_{a b} \mu_{b}, \tilde{\eta}_{a}=Q_{a b} \eta_{b}
$$

expressing $\tilde{\lambda}_{a}, \tilde{\eta}_{a}$ in terms of new variables $\mu_{b}, \eta_{b}$; these forms guarantee that both momentum and super-momentum are conserved, since e.g. $\lambda_{a} \tilde{\lambda}_{a}=\lambda_{a} Q_{a b} \mu_{b}=0$. There is a natural choice for the normalization $q_{b}$ so that the $\mu_{b}, \eta_{b}$ have nice little-group transformation properties under $\lambda_{b} \rightarrow t_{b} \lambda_{b}, \tilde{\lambda}_{b} \rightarrow t_{b}^{1} \tilde{\lambda}_{b}, \tilde{\eta}_{b} \rightarrow t_{b}^{-1} \tilde{\eta}_{b}$. Choosing $q_{b}=\frac{1}{\langle b-1 b\rangle\langle b b+1\rangle}$, $\left(\mu_{b}, \eta_{b}\right) \rightarrow t_{b}\left(\mu_{b}, \eta_{b}\right)$ have exactly the same little group transformation properties as $\lambda_{b}$. Thus we choose

$$
Q_{a b}=\frac{\langle b-1 b+1\rangle \delta_{a b}+\langle b b-1\rangle \delta_{a, b+1}+\langle b+1 b\rangle \delta_{a, b-1}}{\langle b+1 b\rangle\langle b b-1\rangle}
$$

With this choice for $Q$ the relationship between the $D$ minors and $C$ minors becomes

$$
\left(D_{2} \cdots D_{k-1}\right)=\frac{1}{\langle 12\rangle\langle 23\rangle \cdots\langle k-1 k\rangle}\left(C_{1} \cdots C_{k}\right)
$$

where we have given the $\lambda$ dependent factor explicitly. Note also that with this choice

$$
Q_{a b}=Q_{b a}
$$


Now, we would clearly like to change variables from $(k-2) \times n$ variables $C_{\hat{\alpha} a}$ to another set of $(k-2) \times n$ variables $D_{\hat{\alpha} b}=C_{\hat{\alpha} a} Q_{a b}$. Not only are the $k \times k$ minors of $C$ equal (up to $\lambda$ dependent factors) to $(k-2) \times(k-2)$ minors of $D$, but also the $\delta$ functions involving $\tilde{\lambda}$ and $\tilde{\eta}$ are directly functions of $D$, since

$$
\delta^{2}\left(C_{\hat{\alpha} a} \tilde{\lambda}_{a}\right)=\delta^{2}\left(D_{\hat{\alpha} b} \mu_{b}\right), \delta^{4}\left(C_{\hat{\alpha} a} \tilde{\eta}_{a}\right)=\delta^{4}\left(D_{\hat{\alpha} b} \eta_{b}\right)
$$

But we can't literally make this change of variables, since $Q_{a b}$ is not invertible, given that $\lambda_{a} Q_{a b}=0$. Any two $C_{\hat{\alpha} a}$, which differ by any translation in the $\lambda 2$-plane, will yield the same $D$. However, this translational freedom $T_{2}$ was precisely what we gauge-fixed by including the delta function $\prod_{\hat{\alpha}} \delta^{2}\left(C_{\hat{\alpha} a} \lambda_{a}\right)$. Thus, given some fixed $D_{\hat{\alpha} a}$, if there is any solution of $D_{\hat{\alpha} b}=C_{\hat{\alpha} a} Q_{a b}$ satisfying the $2 \times(k-2)$ constraints $C_{\hat{\alpha} a} \lambda_{a}=0$, this solution is unique. On the other hand to be able to find any solution $C_{\hat{\alpha} a}$ to $D_{\hat{\alpha} b}=C_{\hat{\alpha} a} Q_{a b}, D$ can not be a completely general $(k-2) \times n$ matrix, but must satisfy $2 \times(k-2)$ constraints. Since $Q_{a b}=Q_{b a}$ and so $Q_{a b} \lambda_{b}=0$, these constraints are clearly $D_{\hat{\alpha} b} \lambda_{b}=C_{\hat{\alpha} a} Q_{a b} \lambda_{b}=0$. We therefore conclude that

$$
\int d^{(k-2) \times n} C_{\hat{\alpha} a} \prod_{\hat{\alpha}} \delta^{2}\left(C_{\hat{\alpha} a} \lambda_{a}\right) f\left(C_{\alpha a} Q_{a b}\right)=J^{\prime \prime} \int d^{(k-2) \times n} D_{\hat{\alpha} b} \prod_{\hat{\alpha}} \delta^{2}\left(D_{\hat{\alpha} b} \lambda_{b}\right) f\left(D_{\hat{\alpha} b}\right)
$$

here $J^{\prime \prime}$ is yet another Jacobian that only depends on the $\lambda$ 's. We can arrive at this result more formally by beginning with the LHS of eq. (26), and multiply by 1 in the form

$$
1=\int d^{(k-2) \times n} D_{\hat{\alpha} b} \prod_{\hat{\alpha}, b} \delta\left(D_{\hat{\alpha} b}-C_{\hat{\alpha} a} Q_{a b}\right)
$$

We can then perform the integrals over $C$. The $\delta^{2}(C \cdot \lambda)$ factors together with all but $2 \times(k-2)$ of the delta functions introduced in eqn (27) allow us to uniquely solve for all the $C$ 's, leaving us with a remaining $2 \times(k-2) \delta$ functions linear in the $D$ 's, which we know must impose $2 \times(k-2)$ constraints $D \cdot \lambda=0$. This yields the RHS of eq. (26) up to a $\lambda$ dependent Jacobian factor $J^{\prime}$.

We are now done: using eqs. (11), (17), (26) we find

$$
\mathcal{L}_{n ; k}=J^{\prime \prime \prime} \delta^{4}\left(\lambda_{a} \tilde{\lambda}_{a}\right) \delta^{8}\left(\lambda_{a} \tilde{\eta}_{a}\right) \frac{1}{\operatorname{vol}(\mathrm{GL}(k-2))} \int \frac{d^{(k-2) \times n} D_{\hat{\alpha} b}}{\left(D_{1} D_{2} \cdots D_{k-2}\right) \cdots\left(D_{n} D_{1} \cdots D_{k-3}\right)} \delta^{4 \mid 4}\left(D_{\hat{\alpha} b} \mathcal{Z}_{b}^{D}\right)
$$

where $J^{\prime \prime \prime}$ is the product of all the various $\lambda$-dependent prefactors and Jacobians above, and $\mathcal{Z}_{b}^{D}$ are precisely the "momentum twistors" defined by Hodges [5]

$$
\mathcal{Z}^{D}=\left(\begin{array}{c}
\lambda \\
\mu \\
\eta
\end{array}\right)
$$

related to the original momentum space variables via eqs. (21), (22):

$$
\begin{aligned}
& \tilde{\lambda}_{b}=\frac{\langle b+1 b\rangle \mu_{b-1}+\langle b-1 b+1\rangle \mu_{b}+\langle b b-1\rangle \mu_{b+1}}{\langle b-1 b\rangle\langle b b+1\rangle} \\
& \tilde{\eta}_{b}=\frac{\langle b+1 b\rangle \eta_{b-1}+\langle b-1 b+1\rangle \eta_{b}+\langle b b-1\rangle \eta_{b+1}}{\langle b-1 b\rangle\langle b b+1\rangle}
\end{aligned}
$$


We see that the linear operator $Q_{a b}$ so naturally motivated by the geometrical considerations of reducing our initial integral over $G(k, n)$ to the natural integral over $G(k-2, n)$ precisely performs the change of variable from spinor-helicity variables to momentum twistors!

The overall $\lambda$-dependent factor $J^{\prime \prime \prime}$ can simply be determined by explicit computation

$$
J^{\prime \prime \prime}=\frac{1}{\langle 12\rangle\langle 23\rangle \cdots\langle n 1\rangle}
$$

which nicely completes the $\delta$ function prefactors into the full MHV amplitude superamplitude:

$$
\mathcal{L}_{n ; k}=\frac{\delta^{4}\left(\lambda_{a} \tilde{\lambda}_{a}\right) \delta^{8}\left(\lambda_{a} \tilde{\eta}_{a}\right)}{\langle 12\rangle \cdots\langle n 1\rangle} \times \mathcal{R}_{n ; k}
$$

with

$$
\mathcal{R}_{n ; k}=\frac{1}{\operatorname{vol}(\mathrm{GL}(k-2))} \int \frac{d^{(k-2) \times n} D_{\hat{\alpha} b}}{\left(D_{1} D_{2} \cdots D_{k-2}\right) \cdots\left(D_{n} D_{1} \cdots D_{k-3}\right)} \delta^{4 \mid 4}\left(D_{\hat{\alpha} b} \mathcal{Z}_{b}^{D}\right)
$$

This formula for $\mathcal{R}_{n ; k}$ is precisely the one recently presented by Mason and Skinner [6], and makes the dual superconformal invariance of $\mathcal{R}_{n ; k}$ completely manifest. Note that after $\mathrm{GL}(k-2)$ gauge fixing and using the $4 k$ bosonic delta functions, we are left with an integral over $(k-2)(n-k-2)$ variables, which is of course precisely the same as the momentumspace integrals studied in [1]. The residues studied in [1] are also trivially related to those of eq. (34): setting the minor $\left(C_{i} C_{i+1} \ldots C_{i+k-1}\right)$ to zero in [1] corresponds to setting the minor $\left(D_{i+1} D_{i+2} \cdots D_{i+k-2}\right)$ to zero in eq. (34). Therefore, all the results obtained in [1], identifying the various contours associated with tree amplitudes as well as 1- and 2-loop leading singularities, are trivially translated to the corresponding contours for $\mathcal{R}_{n ; k}$ as well.

It is remarkable that the original formula eq. (1) in the usual twistor space is almost identical in form to eq. (33) in the dual momentum-twistor space, differing only by factoring out the MHV superamplitude and shifting $k \rightarrow k-2$. This similarity implies an infinite chain of interesting dualities: we can take the momentum-twistor formula in $G(k-2, n)$ and go to its dual space, factoring out the MHV superamplitude to get an expression in $G(k-4, n)$. In this way we can relate an amplitude with some given $k$ to one with $k \rightarrow k-2 \rightarrow k-4 \cdots$. This shows that in some sense, amplitudes with even (odd) $k$ can be related to MHV (NMHV) amplitudes in some dual space. Furthermore, due to parity we can also send $k \rightarrow n-k$ at any stage in this process and increase $k$ as well, yielding an infinite number of dual descriptions.

We have seen that the $G(k, n)$ integral of eq. (1) simultaneously manifests the superconformal and dual superconformal symmetries, as well as cyclic and parity invariance. It is amusing that different "gauge-fixings" of the GL( $k)$ symmetry are convenient for seeing different symmetries: the gauge-fixing introduced in [1] is more convenient for manifesting parity, while the one exploited in this paper is better suited to manifesting dual superconformal invariance. Since the full Yangian algebra of $\mathcal{N}=4$ SYM scattering amplitudes [8] arises from taking commutators of superconformal and dual superconformal generators, we expect that the object in eq. (1) is invariant under the full Yangian as well. Indeed, as mentioned in [1], perhaps eq. (1) should be thought of as a generating function for all 
Yangian invariants, with residue theorems simply encoding all the remarkable relations between these invariants. It would be interesting to establish the Yangian invariance of eq. (1) even more directly.

\section{Acknowledgments}

We thank Fernando Alday, Jacob Bourjaily, Jared Kaplan, Juan Maldacena, Edward Witten and especially Andrew Hodges, Lionel Mason and David Skinner for stimulating discussions. N.A.-H. is supported by the DOE under grant DE-FG02-91ER40654, F.C. was supported in part by the NSERC of Canada and MEDT of Ontario.

Open Access. This article is distributed under the terms of the Creative Commons Attribution Noncommercial License which permits any noncommercial use, distribution, and reproduction in any medium, provided the original author(s) and source are credited.

\section{References}

[1] N. Arkani-Hamed, F. Cachazo, C. Cheung and J. Kaplan, A Duality For The S Matrix, arXiv:0907.5418 [SPIRES].

[2] J.M. Drummond, J. Henn, V.A. Smirnov and E. Sokatchev, Magic identities for conformal four-point integrals, JHEP 01 (2007) 064 [hep-th/0607160] [SPIRES].

[3] J.M. Drummond, J. Henn, G.P. Korchemsky and E. Sokatchev, Dual superconformal symmetry of scattering amplitudes in $N=4$ super-Yang-Mills theory, Nucl. Phys. B 828 (2010) 317 [arXiv:0807.1095] [SPIRES].

[4] L.F. Alday and J.M. Maldacena, Gluon scattering amplitudes at strong coupling, JHEP 06 (2007) 064 [arXiv:0705.0303] [SPIRES].

[5] A. Hodges, Eliminating spurious poles from gauge-theoretic amplitudes, arXiv:0905.1473 [SPIRES].

[6] L. Mason and D. Skinner, Dual Superconformal Invariance, Momentum Twistors and Grassmannians, JHEP 11 (2009) 045 [arXiv:0909.0250] [SPIRES].

[7] N. Arkani-Hamed, F. Cachazo, C. Cheung and J. Kaplan, The S-matrix in Twistor Space, arXiv:0903.2110 [SPIRES].

[8] J.M. Drummond, J.M. Henn and J. Plefka, Yangian symmetry of scattering amplitudes in $N=4$ super Yang-Mills theory, JHEP 05 (2009) 046 [arXiv:0902.2987] [SPIRES]. 